\title{
Total Small Bowel Volvulus Complicating Common Incomplete Mesentery, An Exceptional Complication in Adults: About A Case
}

Lhaine Merieme $^{1 *}$, Bzikha Ilham ${ }^{2}$, Ouadii Mouaqit ${ }^{1}$, Benjelloun El Bachir ${ }^{1}$, Abdelmalek Ousadden $^{1}$, Ait Taleb Khalid ${ }^{1}$, El Bouhaddouti Hicham ${ }^{1}$

${ }^{1}$ Department of General Surgery A, CHU Hassan II FES, 30000, Morocco

${ }^{2}$ Department of General Surgery, CHU Hassan II FES, 30000, Morocco

DOI: $10.36347 /$ simcr.2020.v08i03.037

| Received: 15.03.2020 | Accepted: 22.03.2020 | Published: 26.03.2020

*Corresponding author: Lhaine Merieme

\section{Abstract}

Total small bowel volvulus complicating commonin complete mesentery is an arrest of rotation of the primary intestinal loop at $180^{\circ}$. Acute volvulus requires emergency surgery. We describe a case of a 65 year old patient who was admitted with total small bowel volvulus on an incomplete common mesentery, who underwent emergency surgery with favorable postoperative outcome.

Keywords: Total volvulus; abnormal rotation; commonin complete mesentery; small intestine.

Copyright @ 2020: This is an open-access article distributed under the terms of the Creative Commons Attribution license which permits unrestricted use, distribution, and reproduction in any medium for non-commercial use (NonCommercial, or CC-BY-NC) provided the original author and source are credited.

\section{INTRODUCTION}

Intestinal malrotation refers to the partial or complete failure rotation of the midgut around the superior mesenteric vessels during embryonic life. Arrested midgut rotation occurs due to the narrow mesentery base and increases the risk of twisting midgut and it's subsequent obstruction and necrosis [1$5]$.

\section{CASE REPORT}

65 years old male patient admitted to the emergency service with abdominal pain, vomiting and inability to passflatus and stool for $48 \mathrm{~h}$. His medical history was unremarkable besides an anxiety disorder.

Blood pressure was measured at $90 \mathrm{mmHg} / 60$ $\mathrm{mmHg}$, pulse at $110 / \mathrm{min}$ and temperature at $38^{\circ} \mathrm{C}$. The physical examination revealed diffuse abdominal pain and muscular defense in each of the four quadrant, as well as an abdominal distention. Rectal examination revealed formed stool without traces of blood. Air-fluid levels were present on the abdominal X-ray.

Computerized tomography scan showeddilated large and small intestine segments up to $69 \mathrm{~mm}$ in diameter, with air-fluid levels alongside a swirling appearance of the mesentery around the superior mesenteric artery and vein indicating the “whirlpoolsign" (Figure-1).

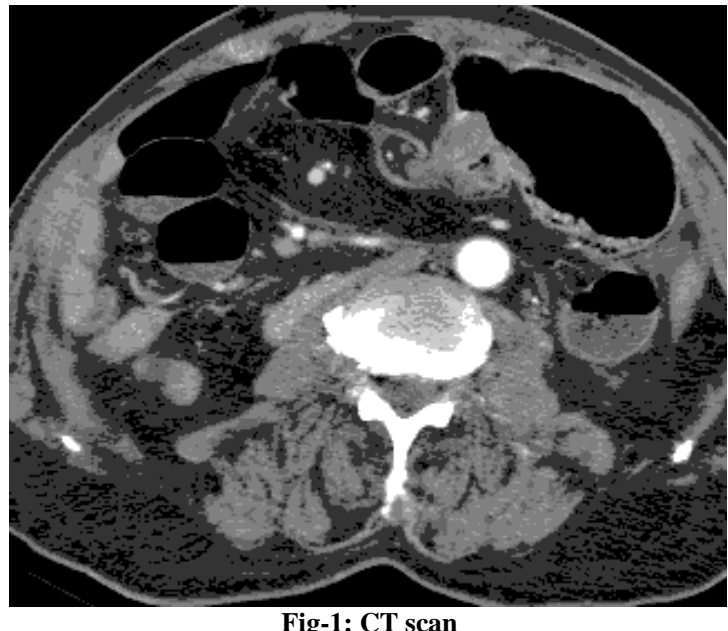

The patient was admitted urgently to the operating room. The exploration found all the distended and suffering hail with a turn concerning the first jejunal loop and the last ilealloop. The caecum is found below the liver and attached to the abdominal wall by a Ladd bridle. The surgical procedure consisted of a counter-clockwise detorsion followed by an immediate recoloration of the small intestine, succeded by the treatment of the embryological rotation anomaly according to the Ladd procedure (section of the flanges, transformation of the incomplete mesentery into complete mesentery in order to prevent recurrence) (Figure-2). Evolution was favorable and the patient was discharged after 7 days of hospitalization. 


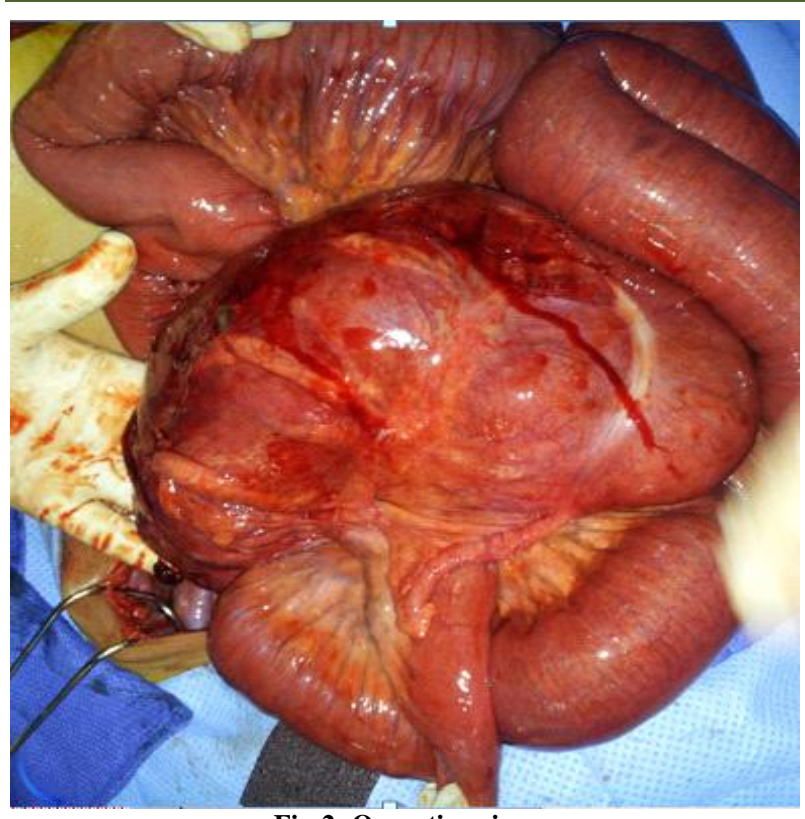

Fig-2: Operative view

\section{DisCUSSION}

Intestinal malrotations are congenital anomalies concerning the rotation and fixation of the intestine and mesentery. During embryonic development, there is normally a rotation of the primary intestinal loop at 270 degrees. Early termination of this rotation isresponsible for the complete (90 degrees) or incomplete (180 degrees) common mesentery. These pathologies are asymptomatic as long as the transit is possible [6]. The prevalence of common mesentery in adults is estimated around $0.2-0.5 \%$ of the population, and this pathology is the main risk factor for small intestine volvulus. Small intestine volvulus is it self a rare disease in adults and is estimated to affect 1.7 in 100,000 persons per year in Western countries [7-9].

Even though clinical symptoms are obscure, adult patients visit hospital mostly with complaints such as vomiting and recurrent abdominal pain, probably due to the chronic partial obstruction. Some may present a malabsorption syndrome due to their ability to eat and protein loss associated with diarrhea caused by chronic volvulus [10].

Acute volvulus requires emergency surgery; imaging must not delay surgery. Ultrasonography and computerized tomography scan can help diagnose malrotation. Contrast enhanced radiography has been shown to be the most accurate method. Typical radiological sign is the corkscrew sign, which is caused by the dilatation of various duodenal segments at different levels and the relocation of duodeno-jejunal junction due to jejunum folding. In ultrasonography, the superior mesenteric vein (SMV) lies to the left or is anterior to the superior mesenteric artery (SMA). Doppler USG may show the Whirlpool sign with rotation of SMV around SMA which is typical for malrotation. Another diagnostic sign of malrotation is when the jejunal arteries lie to the right instead of to the left in computerized tomography scan $[11,12]$.

Surgery is based on the untwisting of the volvulus (counterclockwise) after the assessment of intestinal viability. The intestine placed in the complete common mesentery position: the caecum is situated in the right iliac region [13].

The Ladd procedure allows the incomplete common mesentery to be treated by repositioning it as a complete 90 degree common mesentery. This involves positioning the entire small intestine in the right hemiabdomen and the entire colon in the left hemiabdomen [14].

\section{CONCLUSiON}

The total volvulus of the small intestines on incomplete mesentery having an unspecific symptomatology, makesit essential to evoke this diagnos is early, in order to confirmit, ideally preoperatively by a tomodensitometric examination with injection. In default, any surgeon must at least know how to diagnose on an open abdomen the total volvulus of the small bowel on incomplete mesentery, it's complications and know the principles of it'streatment, according to the procedure of Ladd.

\section{REFERENCES}

1. Lee NK, Kim S, Jeon TY, Kim HS, Kim DH, Seo HI, Park DY, Jang HJ. Complications of congenital and developmental abnormalities of the gastrointestinal tract in adolescents and adults: evaluation with multimodality imaging. Radiographics. 2010 Oct;30(6):1489-507.

2. Plouard C, Rivoal E, Broussine L, Blondin G, Trellu X. Volvulus du grêle sur mésentère commun: intérêt de l'échographie doppler. À propos d'un cas. J Radiol. 2000;81(2):151-3.

3. Oudou AZ, Soumana ID, Souiki T, Majdoub K, Toughrai I, Laalim SA, Mazaz K. Total small bowel volvulus complicating common incomplete mesentery, an exceptional complication in adults: about a case. The Pan African medical journal. 2019;33:220.

4. Gamblin TC, Stephens Jr RE, Johnson RK, Rothwell M. Adult malrotation: a case report and review of the literature. Current surgery. 2003 Sep 1;60(5):517-20.

5. Yousefzadeh DK. The position of the duodenojejunal junction: the wrong horse to bet on in diagnosing or excluding malrotation. Pediatric radiology. 2009 Apr 1;39(2):172-7.

6. Fukuya T, Brown BP, Lu CC. Midgut volvulus as a complication of intestinal malrotation in adults. Digestive diseases and sciences. 1993 Mar $1 ; 38(3): 438-44$.

7. Jain SK. Hirschsprung's disease with intestinal malrotation and midgut volvulus: a rare 
association. Indian J Gastroenterol. 1989;8(3):201.

8. Bodard E, Monheim P, Machiels F, Mortelmans LL. CT of midgutmalrotationpresenting in an adult. Journal Comput Assist Tomogr, 1994;18(3):501-502.

9. Konings-Beetstra EI, van der Jagt EJ. Malrotation of the midgut: a rare complication in an adult patient. Europian Journal Radiol, 1990;11(1):73-7.

10. Claret IC, Agra BC, Taracena BD. Congenital megacolon associated with a common mesentery. Acta pediatrica espanola. 1956 Dec;14(168):108696.

11. Amaral L, Quintanilha R, Bernardo L, Eloi T, Bento F, Santos V. Intestinal malrotation in the elderly. The American Surgeon. 2009 Jul
1;75(7):631-3.

12. Ferguson L, Higgs Z, Brown S, McCarter D, McKay C. Intestinal volvulus following laparoscopic surgery: a literature review and case report. Journal of Laparoendoscopic \& Advanced Surgical Techniques. 2008 Jun 1;18(3):405-10.

13. Geoghegan JG, Maher M, McNally OM, Kirwan WO. Acute midgut volvulus following laparoscopic cholecystectomy. Surgical endoscopy. 1994 Aug 1;8(8):903.

14. Tan V, Kotobi H, Parc Y. Technique chirurgicale: la procédure de Ladd pour volvulus total du grêle sur malrotation. Journal de Chirurgie Viscérale. 2017 Jun 1;154(3):204-8. 